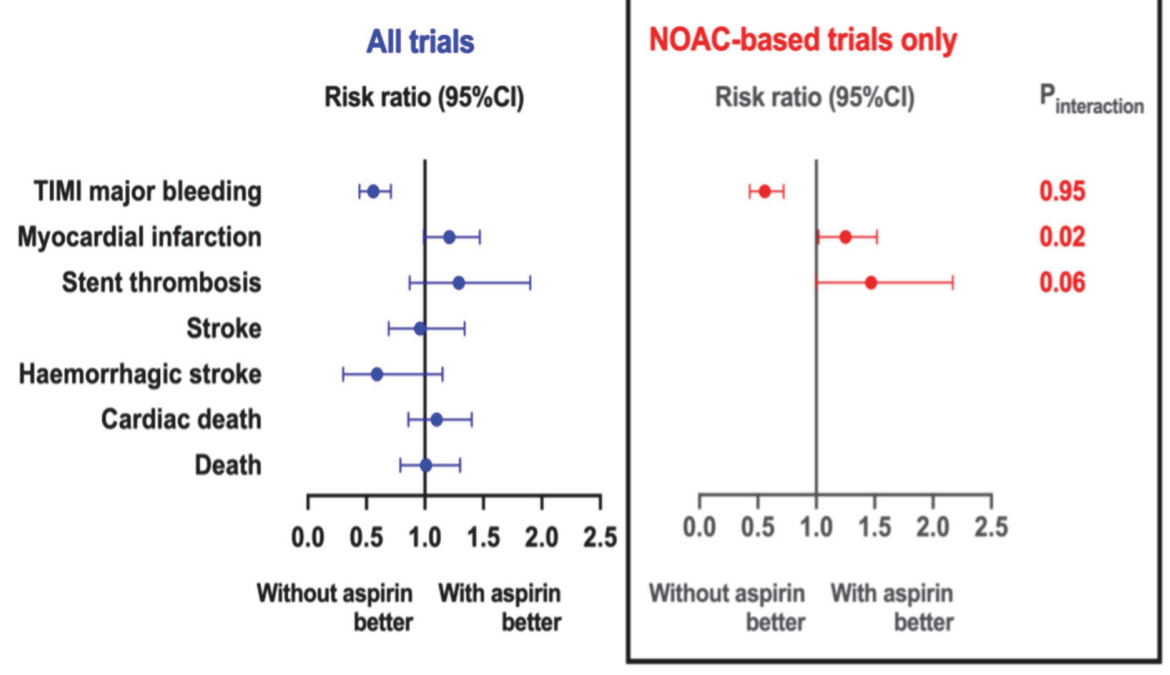

Abstract 14 Figure 1 Risk ratio

\section{DRUG-COATED BALLOON ANGIOPLASTY VERSUS DRUG- ELUTING STENT IMPLANTATION FOR THE TREATMENT OF BARE-METAL AND DRUG-ELUTING STENT RESTENOSIS: RESULTS FROM THE DAEDALUS STUDY}

${ }^{1} \mathrm{D}$ Giacoppo, ${ }^{2} \mathrm{~F}$ Alfonso, ${ }^{3} \mathrm{~A}$ Kastrati, ${ }^{1,4}$ RA Byrne. ${ }^{1}$ Cardiovascular Research Institute (CVRI) Dublin, Mater Private Hospital, Dublin, Ireland; ${ }^{2}$ Department of Cardiology, Hospital Universitario de La Princesa, Madrid, Spain; ${ }^{3}$ Department of Cardiovascular Diseases, German Heart Centre, Munich, Germany; ${ }^{4}$ School of Pharmacy and Bimolecular Sciences, RCSI University of Medicine and Health Sciences, Dublin, Ireland

\subsection{6/heartjnl-2020-ICS.15}

Background In patients with coronary in-stent restenosis (ISR) requiring re-intervention, it is unclear if the choice of treatment strategy depends on whether the restenotic stent was a bare-metal stent (BMS) or a drug-eluting stent (DES).

Objectives We aimed to assess the comparative efficacy and safety of the two most frequently used treatments - angioplasty with drug-coated balloon (DCB) and repeat stenting DES - in patients with BMS- and DES-ISR.

Methods The DAEDALUS study was a pooled analysis of individual patient data from all 10 existing randomized clinical trials comparing DCB angioplasty vs. repeat DES implantation for the treatment of coronary ISR. In this prespecified analysis, patients were stratified according to BMS- vs. DES-ISR, and treatment assigned. The primary efficacy endpoint was target lesion revascularization (TLR) at 3 years. The primary safety endpoint was a composite of all-cause death, myocardial infarction, or target lesion thrombosis at 3 years. Primary analysis was performed by Cox mixed-effects models accounting for the trial of origin. Secondary analyses included nonparsimonious multivariable adjustment accounting also for multiple lesions per patient and two-stage analyses.

Results A total of 710 patients with BMS-ISR (722 lesions) and 1248 with DES-ISR (1377 lesions) were included. In patients with BMS-ISR, no significant difference between treatments was observed in terms of primary efficacy $(9.2 \%$ vs. $10.2 \%$; HR 0.83 , 95\% CI $0.51-1.37$ ) and safety endpoints $(8.7 \%$ vs. $7.5 \%$; HR 1.13 , 95\% CI $0.65-1.96)$; results of secondary analyses were consistent. In patients with DESISR, the risk of the primary efficacy endpoint was higher with DCB angioplasty compared with repeat DES implantation (20.3\% vs. 13.4\%; HR 1.58, 95\% CI 1.16-2.13), while the risk of the primary safety endpoint was only numerically lower (9.5\% vs. 13.3\%; HR 0.69, 95\% CI 0.47-1.00) and interaction was not significant $(p=0.146)$; results of secondary analyses were consistent. The risk of TLR was lower in BMS- vs. DES-ISR $(9.7 \%$ vs. $17.0 \%$; HR 0.57 , 95\% CI $0.42-0.74)$, while safety was not significantly different between ISR types.

Conclusions At 3-year follow-up, DCB angioplasty and repeat stenting with DES are similarly effective and safe in the treatment of BMS-ISR, while DCB angioplasty is significantly less effective than repeat DES implantation in the treatment DESISR without statistically significant differences in safety endpoints. Overall, DES-ISR is associated with higher rates of treatment failure and similar safety compared with BMS-ISR.

\section{Oral abstract presentations 3}

\section{BLOOD PRESSURE REDUCTION AFTER CATHETER-BASED RENAL DENERVATION IN THE ABSENCE OF ANTIHYPERTENSIVE MEDICATIONS: SPYRAL HTN-OFF MED PIVOTAL TRIAL RESULTS}

F Sharif, B McGrath, T O'Connor, M Boehm, K Fario, D Kandzari, F Mahfoud, R Cusack. Galway University Hospital, Galway, Ireland

\subsection{6/heartjnl-2020-ICS.16}

Objective The SPYRAL HTN-OFF MED Pivotal Trial was designed to assess the efficacy of renal denervation (RDN) to lower blood pressure (BP) in the absence of anti-hypertensive medications.

Methods In this international, randomized, sham-controlled trial, patients with hypertension and office systolic blood pressure $(\mathrm{SBP}) \geq 150 \mathrm{mmHg}$ and $<180 \mathrm{mmHg}$ were randomised $1: 1$ to $\mathrm{RDN}$ or sham control. Patients were required to abstain from anti-hypertensive medications prior to randomization. The primary efficacy endpoint was baseline-adjusted change in 24-hour SBP from baseline to 3 months. The secondary efficacy endpoint was baseline-adjusted change in office SBP from baseline to 3 months. Bayesian statistics were used to utilize evidence from the pilot and pivotal studies. 
Results Patients were enrolled at 44 centres in 9 countries. A total of 331 patients were randomised to RDN $(\mathrm{N}=166)$ and sham control $(\mathrm{N}=165)$. The primary and secondary efficacy endpoints were met, with Bayesian posterior probability of superiority $>0.999$. The treatment differences were -3.9 $\mathrm{mmHg}$ for 24-hour SBP and $-6.5 \mathrm{mmHg}$ for office SBP between RDN and sham control groups. Treatment differences in 24-hour and office diastolic pressure at 3 months were -3.1 $\mathrm{mmHg}$ and $-4.4 \mathrm{mmHg}$, respectively. Reductions in BP after RDN were consistent throughout 24 hours. There were no major procedural safety events to 3 months.

Conclusions Results from the SPYRAL HTN-OFF MED Pivotal trial, first reported at ACC2020, demonstrate the efficacy of catheter-based RDN compared to sham control to safely lower $\mathrm{BP}$ in the absence of anti-hypertensive medications.

\section{CORRELATION OF INCIDENTAL LEFT ATRIAL APPENDAGE THROMBUS ON NON-CARDIAC GATED CT WITH TRANSOESOPHAGEAL ECHOCARDIOGRAPHY}

M Waters, C O' Connor, D Moore, B Loo. Tallaght University Hospital, Dublin, Ireland

\subsection{6/heartjnl-2020-ICS.17}

Background Transoesophageal echocardiography is recognized as the gold standard for detection of a left atrial appendage (LAA) thrombus. LAA thrombus is visualized as filling defect on computed tomography (CT), however it can be difficult to differentiate between slow flow and thrombus. This poses challenges in both the reporting and interpretation of left atrial filling defects on non-cardiac gated CTs, and whether to classify this as thrombus or an issue with imaging technique when reporting. Due to the risk of stroke with a LAA thrombus, the reporting of a LAA thrombus on CT makes it difficult for physicians to ignore and usually leads to anticoagulation, thereby exposing the patient to the risk of bleeding. In this study we assessed the correlation between left atrial appendage thrombus or filling defects on a non-cardiac gated CT, with confirmation on TOE.

Methods We retrospectively analyzed TOE cases performed at our center between March 2016 and March 2020. Patients were included if they had been referred for LAA assessment following identification of a suspected LAA thrombus on CT thorax.

Results 757 TOEs were conducted at our center during the study period. This study includes 19 patients who were referred for TOE following incidental left atrial appendage thrombus on CT. Baseline characteristics include; mean age 69.5 years, male $68.4 \%$, hypertensive $79 \%$, history of atrial fibrillation $63.2 \%$, on long-term anticoagulation $63.2 \%$, on anti-coagulation following CT prior to TOE $89.5 \%$, previous stroke 21\%, median CHA2DS2-VASC 3. The mean time from CT to TOE was 16 days.

$15.8 \%(\mathrm{n}=3 / 19)$ of the patients referred had evidence of a LAA thrombus on TOE. $66 \%(n=2)$ of these patients had a history of prior stroke, the median CHA2DS2-VASC score was 5 and overall $50 \%$ of the patients in our cohort who had a history of stroke had a LAA thrombus at TOE. The mean peak LAA filling velocity was only mildly reduced at 37 $\mathrm{cm} / \mathrm{s}$, and $63 \%(\mathrm{n}=12)$ of LAA were windsock morphology. In the follow-up period from TOE to data collection, there was no reported subsequent strokes in the 19 patients analyzed, mean follow-up time 14 months.
Discussion In patients who have an incidental left atrial thrombus or filling defect reported on non-gated CT thorax, only a minority have a thrombus at TOE and it was no associated with subsequent stroke risk. A history of stroke or CHA2DS2-VASC of $>5$ conferred a higher probability of thrombus being present and we recommend that physicians take this into consideration when deciding on anti-coagulation. The LAA filling velocities were only mildly reduced and the radiographic findings are likely related to CT gating rather than an physiological process in the left atrium. Incidental LAA thrombus on CT correlates poorly with presence of thrombus on TOE, and if there is concern a TOE should be performed to assess for a thrombus.

\section{HEART FAILURE PATIENT AND CAREGIVER NEEDS AND EXPECTATIONS REGARDING SELF-MANAGEMENT VIA DIGITAL HEALTH - THE PASSION-HF PROJECT}

${ }^{1}$ A Palant, ${ }^{1} \mathrm{~B}$ Zippel-Schultz, ${ }^{2} \mathrm{~B}$ Brandts, ${ }^{3} \mathrm{C}$ Eurlings, ${ }^{4} \mathrm{M}$ Barrett, ${ }^{4} \mathrm{M}$ Murphy, ${ }^{4} \mathrm{E}$ Furtado $\mathrm{Da}$ Luz Brzychcyk, ${ }^{5} \mathrm{~L}$ Hill, ${ }^{6} \mathrm{~L}$ Dixon, ${ }^{5} \mathrm{D}$ Fitzsimons, ${ }^{5} \mathrm{CF}$ Ski, ${ }^{5} \mathrm{DR}$ Thompson, ${ }^{5} \mathrm{C}$ Watson, ${ }^{2} \mathrm{D}$ Müller-Wieland, ${ }^{2} \mathrm{KA}$ Schuett, ${ }^{7} \mathrm{~T}$ Hoedemakers, ${ }^{3} \mathrm{HP}$ Brunner La-Rocca, ${ }^{3} \mathrm{TM}$ Helms. ${ }^{1}$ German Foundation for the Chronically III, Fürth, Germany; ${ }^{2}$ Department of Cardiology, University Hospital Aachen, Germany; ${ }^{3}$ Department of Cardiology, Maastricht University Medical Center, The Netherlands; ${ }^{4}$ University College Dublin, Mater Misericordiae University Hospital, Dublin, Ireland; ${ }^{5}$ Queen's University Belfast, 97 Lisburn Rd, Belfast, UK; ${ }^{6}$ Belfast Health and Social Care Trust, Belfast City Hospital, Belfast, UK; ${ }^{7}$ Sananet Care BV, Sittard, Netherlands

\subsection{6/heartjn|-2020-ICS.18}

Background Current heart failure (HF) healthcare services are not sufficient to meet the needs of an aging population with increasing comorbidities and disease complexity together with the unequal distribution of medical care in rural and urban regions. These factors have created an imminent need to identify alternative healthcare approaches. eHealth applications have potential to alleviate much of the burden on healthcare services and improve patient treatment. The 'PASSION-HF' project aims to develop a digital decision support system - a virtual doctor - that provides solutions based on current clinical guidelines. Patient independence is maximized through 24/ 7 access to personalized HF management. Furthermore, the application (named DoctorMe) defines processes and decision points where medical professionals need to be included in the process.

Purpose To understand the needs and requirements of HF patients and their informal caregivers in regard to a virtual doctor.

Methods We conducted an exploratory mixed-method study within the Netherlands, United Kingdom, Ireland and Germany. Qualitative, guided interviews were supplemented by a standardized questionnaire. The interviews focused on acceptance factors, motivation to use a decision support system and satisfaction with their current health care situation. Interviews were analysed using the content analysis according to Mayring (2010) with the help of 'Atlas.TI' software. Supplementary questionnaires on self-management of HF-patients, the role of the informal caregivers, technology acceptance, and decision making.

Results A total of 49 patients and 33 informal caregivers were interviewed. Patients were male (76\%) aged between 60 and 69 years $(43 \%)$. Three key themes were identified in regard to digital health: 1) Reassurance: patients often feel uncertain about their condition and their symptoms and a need for 